\title{
Structural and Functional Analysis of the Complex between Citrate and the Zinc Peptidase Carboxypeptidase A
}

\author{
Daniel Fernández, ${ }^{1,2}$ Ester Boix, ${ }^{1}$ Irantzu Pallarès, ${ }^{1,2}$ \\ Francesc X. Avilés, ${ }^{1,2}$ and Josep Vendrell, ${ }^{1,2}$ \\ ${ }^{1}$ Departament de Bioquímica i Biologia Molecular, Facultat de Biociències, Universitat Autònoma de Barcelona,
O8193 Bellaterra, Spain
${ }^{2}$ Institut de Biotecnologia i de Biomedicina, Universitat Autònoma de Barcelona, 08193 Bellaterra, Spain
}

Correspondence should be addressed to Ester Boix, ester.boix@uab.es and Josep Vendrell, josep.vendrell@uab.cat

Received 5 April 2011; Accepted 30 May 2011

Academic Editor: John J. Tanner

Copyright (c) 2011 Daniel Fernández et al. This is an open access article distributed under the Creative Commons Attribution License, which permits unrestricted use, distribution, and reproduction in any medium, provided the original work is properly cited.

A high-resolution carboxypeptidase- $\mathrm{Zn}^{2+}$-citrate complex was studied by X-ray diffraction and enzyme kinetics for the first time. The citrate molecule acts as a competitive inhibitor of this benchmark zinc-dependent peptidase, chelating the catalytic zinc ion in the active site of the enzyme and inducing a conformational change such that carboxypeptidase adopts the conformation expected to occur by substrate binding. Citrate adopts an extended conformation with half of the molecule facing the zinc ion, while the other half is docked in the S1' hydrophobic specificity pocket of the enzyme, in contrast with the binding mode expected for a substrate like phenylalanine or a peptidomimetic inhibitor like benzylsuccinic acid. Combined structural and enzymatic analysis describes the characteristics of the binding of this ligand that, acting against physiologically relevant zinc-dependent proteases, may serve as a general model in the design of new drug-protecting molecules for the oral delivery of drugs of peptide origin.

\section{Introduction}

Carboxypeptidase A (CPA) is the canonical form of the M14 family of peptidases, an important group of physiologically relevant enzymes [1]. M14 peptidases such as CPA and $\mathrm{CPB}$, synthesized in the pancreas and secreted into the gastrointestinal tract, are the major zinc-dependent enzymes responsible for the release of the C-terminal amino acid residue from food protein [2]. Carboxypeptidase activity in the gastrointestinal tract may be modulated by dietary zinc $[3,4]$ or can be inhibited by some organic food components [5-7]. Also, changes in the genomic expression levels may control carboxypeptidase enzyme activity in digestion [8]. In other compartments of the body, different CPs participate in tightly regulated processes such as, for instance, the maturation of physiologically active peptide hormones. Moreover, M14 peptidases are therapeutically interesting molecules because of their involvement in acute pancreatitis [9-11], cancer [12-14], diabetes [15, 16], fibrinolysis [17], and inflammation [18].
Carboxypeptidase A structure reveals that the catalytic zinc ion is coordinated by three protein residues and by one water molecule which is displaced upon substrate binding. The substrate is held in the binding pocket through an ion pair between its C-terminal carboxylate group and the guanidinium side chain of Arg145 and two additional hydrogen bonds with Asp144 and Tyr248. The enzymesubstrate interaction is additionally stabilized by the overall hydrophobic character of the binding pocket which reduces competitive solvation by water molecules [19]. The CPA catalytic mechanism has attracted much attention as a model for zinc-dependent proteases and as such routinely investigated [20-25]. In the two most widely accepted mechanistic pathways, the zinc ion would play a fundamental role either by activating the amide carbonyl of the substrate or by creating a zinc-water nucleophile that directly attacks the amide group [26]. Chelation of the zinc ion is an important aspect in the development of chemotherapeutic agents targeted to this class of enzymes $[27,28]$ as well as in the development of new drug protective molecules for the 
administration of pharmacologically important peptide and protein drugs $[29,30]$.

In the course of our investigations on M14 peptidases and organic synthetic inhibitors [31], we obtained a high resolution $\mathrm{CPA}-\mathrm{Zn}^{2+}$-citrate ternary complex structure. Such an interaction can be understood from the metal-chelating properties of the citrate anion as citrate, a major component in snake venom, is believed to inactivate metal proteases in the prey assisting in venom toxin movement [32]. Database searches revealed only a few instances in which a biological macromolecule- $\mathrm{Zn}^{2+}$-citrate system had been studied at the structural level; surprisingly, none of these involve a biologically active zinc-dependent peptidase. It is interesting to note that in a recent investigation citrate inhibition of carboxypeptidase enzymatic activity has been linked to drug permeation-enhancing properties of the carrier molecule [33]. Thus, the high-resolution structural determination of the CPA- $\mathrm{Zn}^{2+}$-citrate ternary complex presented here along with the kinetic evaluation of citrate inhibition of the CPA catalytic mechanism is intended to gain further insights into the interactions between this coligand of pharmaceutical use and the model zinc-dependent intestinal protease.

\section{Methods}

2.1. Protein Crystallization and Structure Solution. Lyophilized bovine pancreas carboxypeptidase A (Sigma, St. Louis, $\mathrm{MO}$ ) was dissolved in buffer 0.02 M Tris $\mathrm{pH} 7.5 / 1.2 \mathrm{M} \mathrm{LiCl}$ and then desalted and concentrated in Centricon centrifugal filter devices (Millipore, Billerica, MA, USA) against $5 \mathrm{mM}$ Tris, $\mathrm{pH}$ 7.5. During a screening to analyze the structures of CPA-organic inhibitor complexes, CPA crystals were grown from a $2 \mu \mathrm{L}: 2 \mu \mathrm{L}$ mixture of enzyme solution $(10 \mathrm{mg} / \mathrm{mL}$ in $0.02 \mathrm{M}$ Tris, $\mathrm{pH} 7.5$ ) and precipitant (20\% PEG 3, 350, $0.2 \mathrm{M} \mathrm{NH}_{4} \mathrm{Cl}, 0.02 \mathrm{M}$ Tris, $\mathrm{pH}$ 7.0) by the vapour diffusion method. Crystals suitable for X-ray diffraction analysis were grown in the sitting drop setting in an incubator at the steady temperature of $289 \mathrm{~K}$. The largest crystals were harvested and transferred to a new $2 \mu \mathrm{L}$ drop containing the reservoir solution plus a millimolar amount of inhibitor solution. As crystal damage was evident, sequential soakings with increasing inhibitor concentrations were performed. Crystals were harvested, briefly bathed in cryoprotectant buffer (i.e., the reservoir solution with added $30 \%$ glycerol), flash frozen in the nitrogen stream, and diffracted. Structure determination was made with the molecular replacement method using native $\mathrm{CPA}$ (PDB entry $2 \mathrm{ctb}$ ) as the model. Extra electron density accounting for three planar carboxylate groups was clearly evident in the region near the catalytic zinc ion. The shape of this density was easily interpreted as a citrate molecule. Sodium citrate, a buffer used to dissolve the inhibitor, would be the probable source of the ligand seen in the electron density maps. No trace of other inhibitor molecules is seen in the maps. The refinement progressed to convergence and reached a good agreement between the model and the experimental data as shown by the statistics listed in Table 1. Protein X-ray diffraction experiments were performed at the X11 and X13 beamlines of the EMBL (European Molecular Biology Laboratory)-
Outstation at DESY (Deutsches Elektronen Synchrotron), Hamburg, Germany. X-ray diffraction data was processed with Automar (Marresearch GmbH, Norderstedt, Germany). The CCP4 suite [34] and programs PHASER [35] and REFMAC [36] were used in different stages of structure solution and refinement. MolProbity [37] and PROCHECK [38] were employed to check the protein coordinates against reference geometric values. Model building and map fitting were made with Coot [39]. All the final figures were prepared with Pymol (http://www.pymol.org/).

2.2. Kinetic Analysis. Benzylsuccinic acid (BzlSA), sodium citrate, and other reagents were from Sigma (St. Louis, MO). AAFP substrate was from Bachem (Bubendorf, Switzerland). The working solutions included various concentrations of each compound and one AAFP substrate concentration, usually at the $K_{m}$ value. The final assayed concentrations were stepwise varied between $1 \mathrm{nM}$ and $10 \mathrm{mM}$ (for BzlSA) and between $10 \mu \mathrm{M}$ and $100 \mathrm{mM}$ (for citrate). One blank well was used as the control without inhibitor, and the experiments were performed at least by duplicate. The enzyme concentration was adjusted for the kinetic measurements to take place within the initial velocity regime. Changes in absorbance due to proteolytic activity were followed continuously in a multimode plate reader Wallac 1420 Victor3 system (PerkinElmer Life Sciences, Waltham, MA, USA). The kinetic measurements were carried out at room temperature using 96-well microtiter plates (Iwaki Co., Japan). Kinetic activity data was then treated with the GraphPad Version 5.0 package (GraphPad Software, San Diego, CA, USA). After ensuring a good agreement between the experimental observations and the inhibition model, the $K_{i}$ value was calculated from the IC50 following the procedure of Cheng and Prussoff [40]. The goodness of fit between the inhibition model used and the experimental observations was excellent as indicated by the $R^{2}$ parameters, 0.9971 and 0.9822 for BzlSA and citrate, respectively, and by the span of the $95 \%$ confidence interval, in which the $K_{i}$ values lie between $0.39-0.67 \mu \mathrm{M}$ and 3.5-7.0 mM for BzlSA and citrate, respectively.

\section{Results and Discussion}

3.1. Analysis of Structural Data. Although citrate anion is widely used as a buffer in crystal structure determinations, out of the few examples of zinc-dependent proteases bound to this ligand that can be found in the RCSB PDB database, only two hits, namely, human procarboxypeptidase B (entry $1 \mathrm{kwm})$ and porcine procarboxypeptidase A (1pca), were retrieved when searching for carboxypeptidases. Neither of these structures shows citrate in coordination with the catalytic zinc ion, presumably because the presence of the prodomain in both zymogen structures hinders the access to the active site of the enzyme, but the citrate anion resides on the protein's surface exposed to external solvent water molecules. This observation suggests that citrate does bind to exosites in digestive immature proteases, but no structural evidence on its ability to bind to the catalytic machinery of a mature enzyme is available yet. A further query to 
TABLE 1: Statistics of data collection and refinement for citrate-bound CPA.

\begin{tabular}{|c|c|}
\hline Parameters & Value \\
\hline Wavelength used during data collection & $0.8123 \AA$ \\
\hline Unit cell constants & $\begin{array}{c}a=40.61 \AA, b=57.01 \AA \\
c=60.61 \AA \\
\alpha=90.0^{\circ}, \beta=102.4^{\circ}, \gamma=90.0^{\circ}\end{array}$ \\
\hline Resolution range & $20.24-1.70 \AA$ \\
\hline Space group & $\mathrm{P} 2_{1}(1 \mathrm{~mol} /$ asymmetric unit $)$ \\
\hline Number of measured reflections & 185,198 \\
\hline Number of unique reflections & 29,594 \\
\hline$R_{\text {merge }}^{\mathrm{a}}($ overall/outermost shell) & $7.8 / 27.9 \%$ \\
\hline Completeness \& multiplicity (overall/outermost shell) & $\begin{array}{c}99.6 / 99.8 \% \\
2.9 / 3.1\end{array}$ \\
\hline$I / \sigma I$ (overall/outermost shell) & $18.1 / 3.5$ \\
\hline Reflections used for refinement (total/test set) & $26,403 / 893$ \\
\hline Crystallographic $R_{\text {factor }}{ }^{\mathrm{b}} / R_{\text {free }}{ }^{\mathrm{c}}$ & $17.8 / 20.5 \%$ \\
\hline \multicolumn{2}{|c|}{ Deviation from ideality } \\
\hline r.m.s.d. bond lengths & $0.009 \AA$ \\
\hline r.m.s.d. bond angles & $1.18^{\circ}$ \\
\hline Number of protein atoms/total atoms & $2,415 / 2,691$ \\
\hline \multicolumn{2}{|c|}{$B$-factor statistics $\left(\AA^{2}\right)$} \\
\hline Overall $B$-factor/Wilson plot $B$-factor & $17.6 / 25.4$ \\
\hline Catalytic domain, main/side chain & $16.2 / 17.0$ \\
\hline $\mathrm{Zn}^{2+}(1 \mathrm{in}$ total $/ 1 \mathrm{~mol}$ per monomer $)$ & 13.6 \\
\hline Citrate atoms (13 in total/1 mol per monomer) & 20.6 \\
\hline Glycerol atoms (36 in total/ 6 mols per monomer) & 35.4 \\
\hline Solvent atoms (214 in total) & 26.3 \\
\hline \multicolumn{2}{|c|}{ Protein geometry ${ }^{\mathrm{d}}$} \\
\hline Ramachandran favored & $96.7 \%$ (294 of 304 residues) \\
\hline Ramachandran allowed & $99.7 \%$ (303 of 304 residues) \\
\hline Ramachandran outliers & $0.3 \%$ ( 1 of 304 residues, Ser-199) \\
\hline Residues with bad bonds/angles & $0.00 / 0.00 \%$ \\
\hline Rotamer outliers & $0.33 \%$ \\
\hline
\end{tabular}

Notes: ${ }^{\mathrm{a}} R_{\text {merge }}=\Sigma_{\mathrm{hkl}} \Sigma_{j=1}$ to $N\left|I_{\mathrm{hkl}}-I_{\mathrm{hkl}},(j)\right| / \Sigma_{\mathrm{hkl}} \Sigma_{j=1}$ to $N_{\mathrm{Ihkl}}(j)$, where $N$ is the redundancy of the data. The outermost shell is $1.75-1.70 \AA$.

${ }^{\mathrm{b}} R_{\text {factor }}=\Sigma_{\mathrm{hkl}}|| F_{\mathrm{obs}}|-| F_{\text {calc }}|| / \Sigma_{\mathrm{hkl}}\left|F_{\mathrm{obs}}\right|$, where the $F_{\mathrm{obs}}$ and $F_{\text {calc }}$ are the observed and calculated structure factor amplitudes of reflection hkl.

${ }^{\mathrm{c}} R_{\text {free }}=$ is equal to $R_{\text {factor }}$ for a randomly selected $3.3 \%$ subset of reflections that were not used in refinement.

${ }^{\mathrm{d}}$ According to Molprobity [37].

the RSCB PDB revealed a total of 13 macromolecule-zinccitrate structures, none of which involve digestive enzymes. Therefore, the present crystal structure determination represents the first example of a metal-dependent, fully active, physiologically relevant protease in a ternary complex with citrate. A crystal belonging to the monoclinic space group $\mathrm{P} 22_{1}$ that diffracted beyond $1.70 \AA$ was characterized in this study; crystallographic and refinement statistics details are presented in Table 1. The atomic coordinates and structure factors have been validated and deposited with the RCSB PDB with accession code 3kgq.

3.2. The Binding of Citrate to the Catalytic Centre in the CPA Active Site. One citrate anion per carboxypeptidase$\mathrm{Zn}^{2+}$ cation was identified from the electron density maps (Figure 1). Citrate binds in an extended conformation to the catalytic centre of the enzyme's active site, where the amide bond is be hydrolyzed, across subsites S1 (defined by residues Arg127 and Glu270, which are involved in the nucleophilic attack of the scissile amide carbon) and $\mathrm{S1}^{\prime}$ (Asn144, Arg145, and Tyr248, which anchor and stabilize the peptide substrate). All but one of the four functional groups from citrate make important interactions with the metal and several ligands from the protein (Table 2 and Figure 2).

The zinc ion is bidentately chelated by citrate oxygen atoms $\mathrm{O} 1$ and $\mathrm{O} 2$, both atoms lying in the proximity of two zinc ligands from the protein (His69 and Glu72). Oxygen atoms from two different carboxylate groups are hydrogen bonded to Arg127. The nucleophile Glu270 forms a strong hydrogen bond to one carboxylate and the hydroxyl group. An ion pair is formed between oxygen atoms O5 and $\mathrm{O} 6$ from citrate and the guanidinium side chain of 


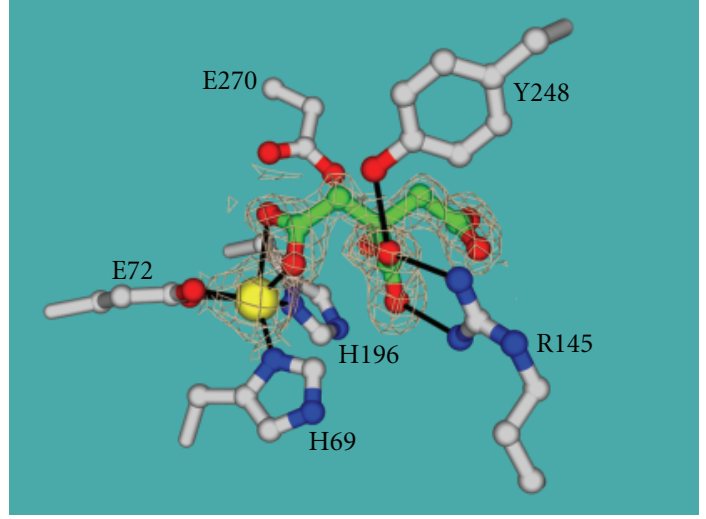

Figure 1: Partial view of the carboxypeptidase- $\mathrm{Zn}^{2+}$-citrate complex structure localized around the citrate ligand. The ligand is coloured green while protein atoms are in grey. Oxygen and nitrogen atoms are red and blue, respectively. The $\mathrm{Zn}^{2+}$ ion is depicted as a sphere in yellow. Relevant residues for binding are shown as sticks and labelled. Polar interactions are shown as solid lines in black. The $2 \mathrm{~F}_{\mathrm{o}}-\mathrm{F}_{\mathrm{c}}$ map, calculated by deleting citrate and $\mathrm{Zn}^{2+}$ coordinates, is contoured at 1.0 sigma (gold mesh).

TABle 2: Geometric details of the interaction between protein residues and the citrate ligand.

\begin{tabular}{|c|c|c|c|}
\hline \multirow{2}{*}{ Protein residue ID } & \multicolumn{2}{|c|}{ Ligand atoms } & \multirow{2}{*}{ Distance $(\AA)$} \\
\hline & Protein & Citrate & \\
\hline \multirow{2}{*}{$\mathrm{Zn}$} & \multirow{2}{*}{$\mathrm{Zn}^{2+}$} & $\mathrm{O} 2$ & 2.1 \\
\hline & & O1 & 2.7 \\
\hline His69 & $\mathrm{N} \delta 1$ & $\mathrm{O} 2$ & 3.0 \\
\hline \multirow{2}{*}{ Glu72 } & $\mathrm{O} \varepsilon 2$ & $\mathrm{O} 2$ & 3.2 \\
\hline & $\mathrm{O} \varepsilon 2$ & O1 & 3.3 \\
\hline \multirow{2}{*}{ Arg127 } & $\mathrm{N} \eta 1$ & $\mathrm{O} 2$ & 3.2 \\
\hline & $\mathrm{N} \eta 2$ & O5 & 3.0 \\
\hline Asn 144 & $\mathrm{~N} \delta 2$ & O6 & 2.9 \\
\hline \multirow{3}{*}{ Arg145 } & $\mathrm{N} \eta 1$ & O5 & 3.2 \\
\hline & $\mathrm{N} \eta 1$ & O6 & 2.8 \\
\hline & $\mathrm{N} \eta 2$ & O5 & 2.7 \\
\hline Ser197 & $\mathrm{O}$ & $\mathrm{O} 1$ & 3.1 \\
\hline Tyr248 & $\mathrm{O} \eta$ & O5 & 2.6 \\
\hline \multirow{3}{*}{ Glu270 } & $\mathrm{O} \varepsilon 1$ & $\mathrm{O} 1$ & 2.4 \\
\hline & $\mathrm{O} \varepsilon 2$ & O1 & 3.0 \\
\hline & $\mathrm{O} \varepsilon 2$ & O7 & 2.8 \\
\hline \multirow{2}{*}{$\mathrm{HOH} 213$} & $\mathrm{O}$ & $\mathrm{O} 4$ & 2.6 \\
\hline & $\mathrm{O}$ & O3 & 3.3 \\
\hline HOH109 & $\mathrm{O}$ & $\mathrm{O} 3$ & 2.6 \\
\hline HOH191 & $\mathrm{O}$ & O1 & 2.9 \\
\hline
\end{tabular}

Arg145. These atoms make additional interactions with the other S1' residues, Asn144 and Tyr248. The latter residue has been identified as a key player in the CPA catalyzed mechanism by site-specific mutagenesis studies [24]. The conformational change suffered by Tyr248 upon ligand binding is well documented: the aromatic ring switches by $120^{\circ}$ its location between the solvent exposed "open" to the

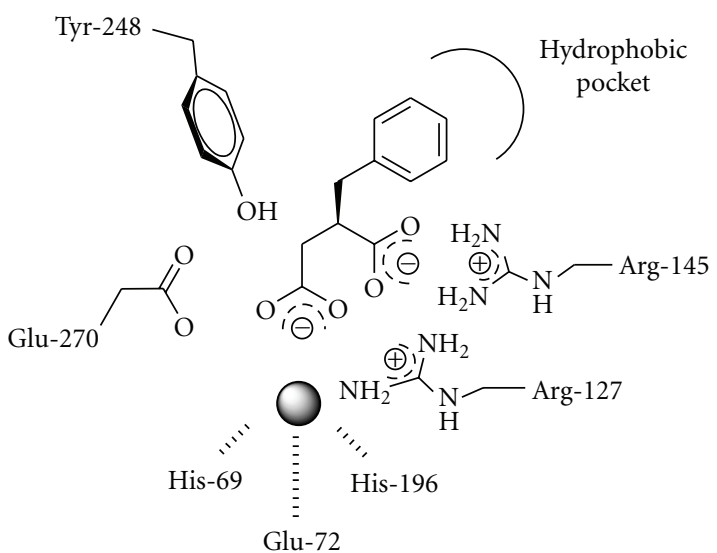

(a)

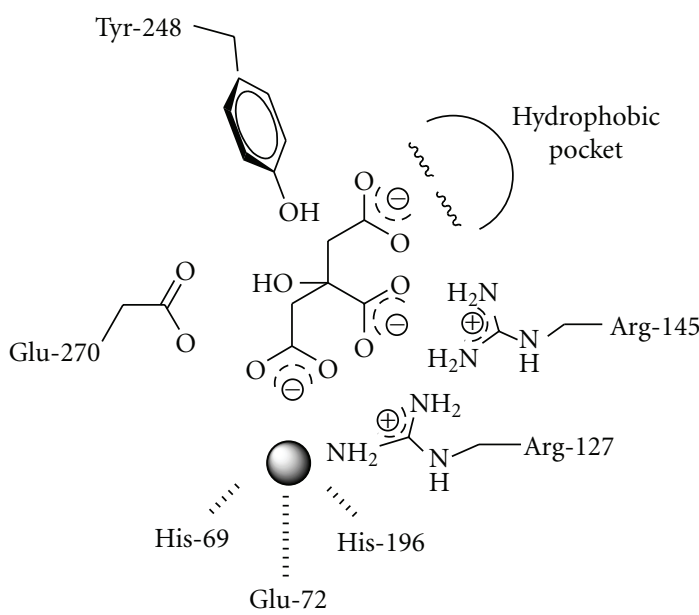

(b)

Figure 2: The structures of CPA bound to the peptidomimetic BzlSA (a) and citrate (b) are schematically shown. Important residues for binding the zinc ion (sphere) are included. Water molecules shielding citrate are shown as wavy lines.

"closed" conformation with the phenolic hydroxyl pointing to the catalytic zinc $[41,42]$. In this paper, Tyr248 is in the closed conformation expected to occur when the active site of the enzyme is occupied. As seen in other CPA-bound structures, the water molecule is displaced from the zinc coordination sphere by a carboxylate from the ligand and the citrate molecule binds to CPA in a manner reminiscent of the succinate-based ligands such as the benchmark inhibitor benzylsuccinic acid (BzlSA). As will be commented next, while the aromatic ring of BzlSA is buried in the hydrophobic pocket of CPA, the carboxylate group of citrate that occupies the corresponding edge of the molecule adapts its conformation to this specific enzyme's environment.

3.3. Citrate Burial in the Hydrophobic Pocket of CPA. Like citrate, BzlSA chelates zinc and forms an ion pair with Arg145 and hydrogen bonds to catalytically key residues like Tyr248, but the two molecules also display considerable differences in binding to the enzyme (Figure 3). From 


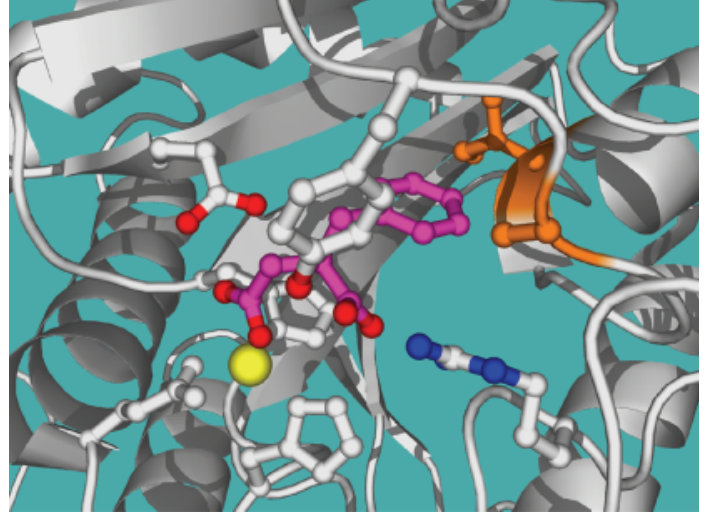

(a)

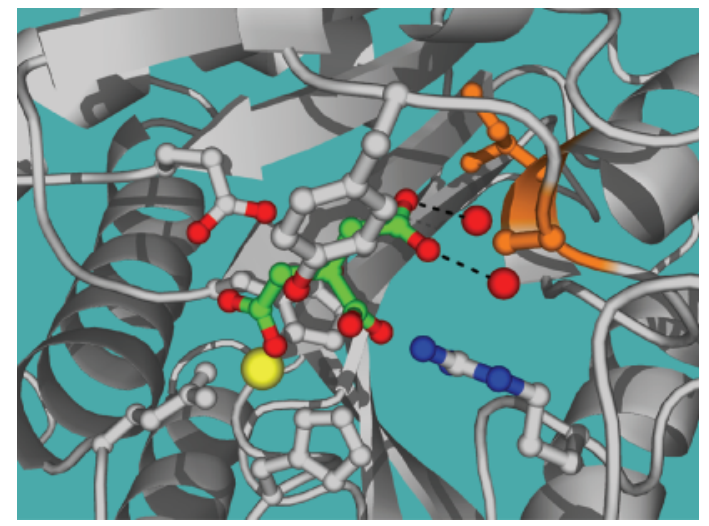

(b)

FIGURE 3: Comparison of carboxypeptidase-bound structures. (a) CPA in complex with BzlSA (PDB entry 1cbx). (b) CPA with bound citrate (this work). BzlSA and citrate are shown magenta and green, respectively. Ala250 and Ile255, enclosing the ligand, are in orange. Note the location of two water molecules (spheres in red) at (b), masking hydrophobic residues at the bottom of the active site. Hydrogen bond interactions are as dashed lines in black. Both structures are shown in similar orientation.

inspection of the crystal structure in complex to $\mathrm{CPA}$ (PDB entry $1 \mathrm{cbx}$ ), BzlSA extends towards the bottom of the active site cleft with an aromatic ring that mimics a phenylalanine residue. Side chains Leu203, Ile243, Ala250, Gly253, Ile255, and Thr268 define the hydrophobic pocket that harbours the specificity-determinant residue in the M14 peptidase family, which for CPA is Ile255. This hydrophobic pocket is best suited to accommodate an aromatic ring, and Ile255 makes hydrophobic interactions with this part of the inhibitor (Figure 3(a)).

The third carboxylate group from citrate is the only one shielded from contact to CPA. Each one of the oxygen atoms of the carboxylate group, $\mathrm{O} 3$ and $\mathrm{O} 4$, makes strong hydrogen bonds to water molecules, which in turn are hydrogen bonded to other water molecules and amino acid side chains. Thus, this ionisable group lies at least about $3.5 \AA$ apart from the wall of the hydrophobic pocket (the closest residue is Ala250) and at more than $7 \AA$ from Ile255. Compared to the BzlSA bound structure, the conformation of the latter residue is substantially shiftedas measured by the $C \alpha-C \beta$ -

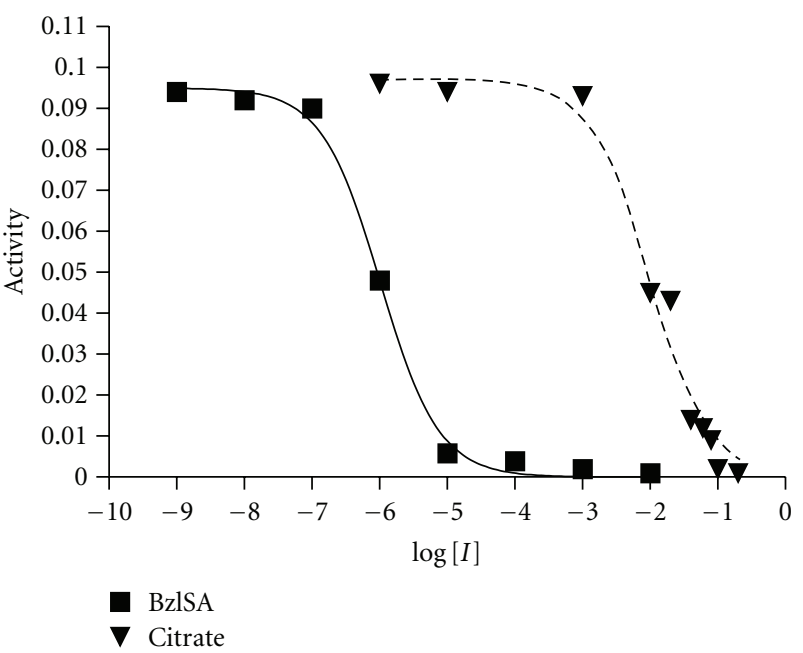

FIGURE 4: Fit of a competitive inhibition model (continuous linesquares, BzlSA; dashed line-triangles, citrate) to the experimental observations.

TABle 3: Kinetic parameters of BzlSA and citrate inhibition.

\begin{tabular}{lcccc}
\hline Compound & $\begin{array}{c}\text { Type of } \\
\text { inhibition }\end{array}$ & Substrate & $K_{i}(\mu \mathrm{M})$ & Reference \\
\hline \multirow{3}{*}{ BzlSA } & competitive & Hipp-Phe $^{\mathrm{a}}$ & 1.1 & {$[43]$} \\
& competitive & O-Cl-Phe & 0.2 & {$[44]$} \\
& competitive & N-Ac-PSP $^{\mathrm{b}}$ & $0.51-0.61$ & {$[49]$} \\
citrate & competitive & AAFP $^{\mathrm{d}}$ & 0.52 & This work \\
\hline
\end{tabular}

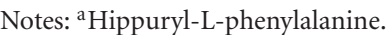

${ }^{\mathrm{b}} \mathrm{O}$-(trans- $p$-chlorocinnamoyl)-L- $\beta$-phenylacetate.

${ }^{c} N$-acetyl-phenylalanyl-3-thiaphenylalanine.

${ }^{\mathrm{d}} \mathrm{N}$-(4-methoxyphenylazoformyl)-L-phenylalanine.

$\mathrm{C} \gamma 1-\mathrm{C} \delta 1$ torsion angle: $160.3^{\circ}$ and $-37.7^{\circ}$ for the BzlSA and citrate CPA-bound forms, respectively. This rearrangement of the Ile 255 side chain would reflect the ability of the enzyme to adapt the hydrophobic pocket to the features of the ligand molecule.

3.4. Kinetic Assays of CPA Ligands. Kinetic activity measurements were carried out to determine the ability of citrate to inhibit the CPA catalyzed reaction. The measurements were made in parallel with BzlSA to provide a direct comparison with this standard inhibitor. The nonlinear global fitting of the experimental data to a competitive inhibition model was excellent (Figure 4). Besides, the shape of the citrate inhibition curve resembles that of BzlSA whose $K_{i}$ is in excellent agreement with values obtained in previous works (Table 3).

From Table 3, it is evident that citrate performs as a very weak CPA inhibition compared to BzlSA, a likely consequence of the presence of the aromatic moiety in the latter. BzlSA was designed as a by-product analogue, that is, it retains features of the cleaved products of the CPA catalytic reaction [43] in such as way that the aromatic 
moiety docks into the hydrophobic pocket of the enzyme, displacing water molecules at the bottom of the active site cleft. This displacement, together with the movement of Tyr248, substantially facilitate the enzymatic activity by creating an overall hydrophobic environment. It is well documented that amino acid substrates and products are very weak CPA inhibitors. For example, L-Phe is, like it has been calculated here for citrate, a $5 \mathrm{mM} \mathrm{CPA}$ inhibitor [43]. Although citrate would be easily displaced by the substrate AAFP when competing for binding to CPA in the in vitro experimental assays, a completely different scenario may be possible in vivo such that citrate could be equally well suited as the natural substrates are to bind to the active site of metaldependent proteases.

\section{Conclusion}

Citric acid has intestinal absorption enhancing effects and is widely used in drug formulations. Recent evidence demonstrated that the citrate salt of chitosan has a drug penetration enhancement and that this effect is linked to the inhibition of metal-dependent enzymes like leucine aminopeptidase (a membrane protease) and the luminal protease CPA [44, 45]. In line with these observations, we report further structural and kinetic evidence of the interaction between CPA and citrate, in which the organic anion chelates the zinc cation at the active site of the enzyme. The citrate mode of binding resembles that of the peptidomimetic substrate analogue benzylsuccinic acid, which also coincides with citrate in being a competitive CPA inhibitor. The $K_{i}$ value for citrate is much weaker than that of benzylsuccinic acid but comparatively similar to that of simple amino acids, suggesting that citrate would compete for the in vivo binding of zinc at the active site of intestinal enzymes.

There is a wide range of digestive protease inhibitors with potential as peptide drug-protecting agents that, like commonly used excipients, may be associated with some drawbacks as elevated costs or risk of toxic side effects $[46,47]$. Efforts to combine a protease inhibitor and a drug permeation enhancer in one single molecule to increase the stability and absorption of peptide or protein drugs are an increasingly investigated matter [48]. As recently reported [33], citrate, a commonly used excipient, may be an example of such type of molecules. The analysis of the newly determined carboxypeptidase- $\mathrm{Zn}^{2+}$-citrate complex structure might help in the structure-guided design of newer drug protecting compounds targeted to metal-dependent proteases.

\section{Acknowledgments}

Financial support from the Ministerio de Ciencia e Innovación, Spain (Grant BIO2007-68046) and Generalitat de Catalunya (Grant 2005SGR-1037) is gratefully acknowledged. The authors wish to express their gratitude to the local staff at the EMBL (European Molecular Biology Laboratory), Outstation at DESY (Deutsches Elektronen Synchrotron), Hamburg, Germany, for their assistance in X-ray data collection.

\section{References}

[1] J. Vendrell, F. X. Aviles, and L. D. Fricker, "Metallocarboxypeptidases," in Handbook of Metalloproteins, A. Messerschmidt, W. Bode, and M. Cygler, Eds., pp. 176-189, John Wiley \& Sons, Chichester, UK, 2004.

[2] R. H. Erickson and Y. S. Kim, "Digestion and absorption of dietary protein," Annual Review of Medicine, vol. 41, pp. 133139, 1990.

[3] J. Berger and B. Olds Schneeman, "Stimulation of bilepancreatic zinc, protein and carboxypeptidase secretion in response to various proteins in the rat," Journal of Nutrition, vol. 116, no. 2, pp. 265-272, 1986.

[4] J. Berger and B. O. Schneeman, "Intestinal zinc and carboxypeptidase A and B activity in response to consumption of test meals containing various proteins by rats," Journal of Nutrition, vol. 118, no. 6, pp. 723-728, 1988.

[5] A. Ibarz, A. Garvín, S. Garza, and J. Pagán, "Toxic effect of melanoidins from glucose-asparagine on trypsin activity," Food and Chemical Toxicology, vol. 47, no. 8, pp. 2071-2075, 2009.

[6] C. J. Martin and W. J. Evans, "Phytic acid-enhanced metal ion exchange reactions: the effect on carboxypeptidase A1," Journal of Inorganic Biochemistry, vol. 35, no. 4, pp. 267-288, 1989.

[7] J. Parellada, G. Suárez, and M. Guinea, "Inhibition of zinc metallopeptidases by flavonoids and related phenolic compounds: structure-activity relationships," Journal of Enzyme Inhibition, vol. 13, no. 5, pp. 347-359, 1998.

[8] M. M. Martínez-Montemayor, G. M. Hill, N. E. Raney et al., "Gene expression profiling in hepatic tissue of newly weaned pigs fed pharmacological zinc and phytase supplemented diets," BMC Genomics, vol. 9, article 421, 2008.

[9] M. A. Hilal, C. T. Ung, S. Westlake, and C. D. Johnson, "Carboxypeptidase-B activation peptide, a marker of pancreatic acinar injury, but not L-selectin, a marker of neutrophil activation, predicts severity of acute pancreatitis," Journal of Gastroenterology and Hepatology, vol. 22, no. 3, pp. 349-354, 2007.

[10] S. Regnér, S. Appelros, C. Hjalmarsson, J. Manjer, J. Sadic, and A. Borgström, "Monocyte chemoattractant protein 1, active carboxypeptidase B and CAPAP at hospital admission are predictive markers for severe acute pancreatitis," Pancreatology, vol. 8, no. 1, pp. 42-49, 2008.

[11] J. Sáez, J. Martínez, C. Trigo et al., "Clinical value of rapid urine trypsinogen-2 test strip, urinary trypsinogen activation peptide, and serum and urinary activation peptide of carboxypeptidase B in acute pancreatitis," World Journal of Gastroenterology, vol. 11, no. 46, pp. 7261-7265, 2005.

[12] S. Matsugi, T. Hamada, N. Shioi, T. Tanaka, T. Kumada, and S. Satomura, "Serum carboxypeptidase A activity as a biomarker for early-stage pancreatic carcinoma," Clinica Chimica Acta, vol. 378, no. 1-2, pp. 147-153, 2007.

[13] F. Fialka, R. M. Gruber, R. Hitt et al., "CPA6, FMO2, LGI1, SIAT1 and TNC are differentially expressed in early- and late-stage oral squamous cell carcinoma-a pilot study," Oral Oncology, vol. 44, no. 10, pp. 941-948, 2008.

[14] P. L. Ross, I. Cheng, X. Liu et al., "Carboxypeptidase 4 gene variants and early-onset intermediate-to-high risk prostate cancer," BMC Cancer, vol. 9, article 69, 2009.

[15] K. D. Jeffrey, E. U. Alejandro, D. S. Luciani et al., "Carboxypeptidase E mediates palmitate-induced $\beta$-cell ER stress and apoptosis," Proceedings of the National Academy of Sciences of the United States of America, vol. 105, no. 24, pp. 8452-8457, 2008. 
[16] J. D. Johnson, "Proteomic identification of carboxypeptidase E connects lipid-induced $\beta$-cell apoptosis and dysfunction in type 2 diabetes," Cell Cycle, vol. 8, no. 1, pp. 38-42, 2009.

[17] D. A. Tregouet, R. Schnabel, M. C. Alessi et al., "Activated thrombin activatable fibrinolysis inhibitor levels are associated with the risk of cardiovascular death in patients with coronary artery disease: the Athero Gene study," Journal of Thrombosis and Haemostasis, vol. 7, no. 1, pp. 49-57, 2009.

[18] L. L. Leung, T. Myles, T. Nishimura, J. J. Song, and W. H. Robinson, "Regulation of tissue inflammation by thrombinactivatable carboxypeptidase B (or TAFI)," Molecular Immunology, vol. 45, no. 16, pp. 4080-4083, 2008.

[19] C. Schmuck, "How to improve guanidinium cations for oxoanion binding in aqueous solution?. The design of artificial peptide receptors," Coordination Chemistry Reviews, vol. 250, no. 23-24, pp. 3053-3067, 2006.

[20] D. M. Hayes and P. A. Kollman, "Electrostatic potentials of proteins. 1. Carboxypeptidase A," Journal of the American Chemical Society, vol. 98, no. 11, pp. 3335-3345, 1976.

[21] L. Banci, I. Bertini, and G. La Penna, "The enzymatic mechanism of carboxypeptidase: a molecular dynamics study," Proteins: Structure, Function and Genetics, vol. 18, no. 2, pp. 186-197, 1994.

[22] K. Zhang, J. Dong, and D. S. Auld, "A time-resolved X-Ray absorption fine structure study of substrate hydrolysis by carboxypeptidase A," Physica B (Amsterdam), vol. 208 \& 209, no. 1-4, pp. 719-721, 1995.

[23] S. Álvarez-Santos, À. González-Lafont, J. M. Lluch, B. Oliva, and F. X. Avilés, "Theoretical study of the role of arginine 127 in the water-promoted mechanism of peptide cleavage by carboxypeptidase A," New Journal of Chemistry, vol. 22, no. 4, pp. 319-325, 1998.

[24] J. H. Cho, D. H. Kim, D. H. Kim, K. J. Lee, and K. Y. Choi, "The role of Tyr248 probed by mutant bovine carboxypeptidase A: insight into the catalytic mechanism of carboxypeptidase A," Biochemistry, vol. 40, no. 34, pp. 10197-10203, 2001.

[25] D. Xu and H. Guo, "Quantum mechanical/molecular mechanical and density functional theory studies of a prototypical zinc peptidase (carboxypeptidase A) suggest a general acid-general base mechanism," Journal of the American Chemical Society, vol. 131, no. 28, pp. 9780-9788, 2009.

[26] D. H. Kim, "Chemistry-based design of inhibitors for carboxypeptidase A," Current Topics in Medicinal Chemistry, vol. 4, no. 12, pp. 1217-1226, 2004.

[27] M. Adler, B. Buckman, J. Bryant et al., "Structures of potent selective peptide mimetics bound to carboxypeptidase B," Acta Crystallographica. Section D: Biological Crystallography, vol. 64, no. 2, pp. 149-157, 2008.

[28] M. E. Bunnage and D. R. Owen, "TAFIa inhibitors in the treatment of thrombosis," Current Opinion in Drug Discovery and Development, vol. 11, no. 4, pp. 480-486, 2008.

[29] I. Bravo-Osuna, C. Vauthier, A. Farabollini, G. Millotti, and G. Ponchel, "Effect of chitosan and thiolated chitosan coating on the inhibition behaviour of PIBCA nanoparticles against intestinal metallopeptidases," Journal of Nanoparticle Research, vol. 10, no. 8, pp. 1293-1301, 2008.

[30] R. Rink, A. Arkema-Meter, I. Baudoin et al., "To protect peptide pharmaceuticals against peptidases," Journal of Pharmacological and Toxicological Methods, vol. 61, no. 2, pp. 210-218, 2010.

[31] D. Fernández, S. Testero, J. Vendrell, F. X. Avilés, and S. Mobashery, "The X-ray structure of carboxypeptidase a inhibited by a thiirane mechanism-based ihibitor," Chemical Biology and Drug Design, vol. 75, no. 1, pp. 29-34, 2010.
[32] G. V. Odell, P. C. Ferry, L. M. Vick et al., "Citrate inhibition of snake venom proteases," Toxicon, vol. 36, no. 12, pp. 1801-1806, 1998.

[33] M. C. Bonferoni, G. Sandri, S. Rossi, F. Ferrari, S. Gibin, and C. Caramella, "Chitosan citrate as multifunctional polymer for vaginal delivery. Evaluation of penetration enhancement and peptidase inhibition properties," European Journal of Pharmaceutical Sciences, vol. 33, no. 2, pp. 166-176, 2008.

[34] Collaborative Computational Project number 4, "The CCP4 suite: programs for protein crystallography," Acta Crystallographica. Section D: Biological Crystallography, vol. 50, pp. 760-776, 1994.

[35] R. J. Read, "Pushing the boundaries of molecular replacement with maximum likelihood," Acta Crystallographica. Section D Biological Crystallography, vol. 57, no. 10, pp. 1373-1382, 2001.

[36] G. N. Murshudov, A. A. Vagin, and E. J. Dodson, "Refinement of macromolecular structures by the maximum-likelihood method," Acta Crystallographica. Section D: Biological Crystallography, vol. 53, no. 3, pp. 240-255, 1997.

[37] I. W. Davis, L. W. Murray, J. S. Richardson, and D. C. Richardson, "MolProbity: structure validation and all-atom contact analysis for nucleic acids and their complexes," Nucleic Acids Research, vol. 32, pp. W615-W619, 2004.

[38] R. A. Laskowski, M. W. MacArthur, E. Hutchinson, and J. M. Thornton, "PROCHECK: a program to check the stereochemical quality of protein structures," Journal of Applied Crystallography, vol. 26, pp. 283-291, 1993.

[39] P. Emsley and K. Cowtan, "Coot: model-building tools for molecular graphics," Acta Crystallographica. Section D: Biological Crystallography, vol. 60, no. 12, pp. 2126-2132, 2004.

[40] Y. Cheng and W. H. Prusoff, "Relationship between the inhibition constant (K1) and the concentration of inhibitor which causes 50 per cent inhibition (I50) of an enzymatic reaction," Biochemical Pharmacology, vol. 22, no. 23, pp. 3099-3108, 1973.

[41] D. Fernández, E. Boix, I. Pallarès, F. X. Avilés, and J. Vendrell, "Analysis of a new crystal form of procarboxypeptidase B: further insights into the catalytic mechanism," Biopolymers, vol. 93, no. 2, pp. 178-185, 2010.

[42] S. Firth-Clark, S. B. Kirton, H. M. G. Willems, and A. Williams, "De novo ligand design to partially flexible active sites: application of the ReFlex algorithm to carboxypeptidase A, acetylcholinesterase, and the estrogen receptor," Journal of Chemical Information and Modeling, vol. 48, no. 2, pp. 296-305, 2008.

[43] L. D. Byers and R. Wolfenden, "A potent reversible inhibitor of carboxypeptidase A," The Journal of Biological Chemistry, vol. 247, no. 2, pp. 606-608, 1972.

[44] R. E. Galardy and Z. P. Kortylewicz, "Inhibition of carboxypeptidase A by aldehyde and ketone substrate analogues," Biochemistry, vol. 23, no. 9, pp. 2083-2087, 1984.

[45] M. C. Bonferoni, G. Sandri, S. Rossi, F. Ferrari, and C. Caramella, "Chitosan and its salts for mucosal and transmucosal delivery," Expert Opinion on Drug Delivery, vol. 6, no. 9, pp. 923-939, 2009.

[46] T. Kean, S. Roth, and M. Thanou, "Trimethylated chitosans as non-viral gene delivery vectors: cytotoxicity and transfection efficiency," Journal of Controlled Release, vol. 103, no. 3, pp. 643-653, 2005.

[47] M. G. Ursino, E. Poluzzi, C. Caramella, and F. De Ponti, "Excipients in medicinal products used in gastroenterology as a possible cause of side effects," Regulatory Toxicology and Pharmacology, vol. 60, no. 1, pp. 93-105, 2011. 
[48] M. D. Del Curto, A. Maroni, L. Palugan, L. Zema, A. Gazzaniga, and M. E. Sangalli, "Oral delivery system for two-pulse colonic release of protein drugs and protease inhibitor/ absorption enhancer compounds," Journal of Pharmaceutical Sciences, vol. 100, no. 8, pp. 3251-3259, 2011.

[49] M. P. Wentland, S. Raza, and Y. Gao, "96-Well plate colorimetric assay for Ki determination of $( \pm)$-2-Benzylsuccinic acid, an inhibitor of carboxypeptidase A. A laboratory experiment in drug discovery," Journal of Chemical Education, vol. 81, no. 3, pp. 398-400, 2004. 

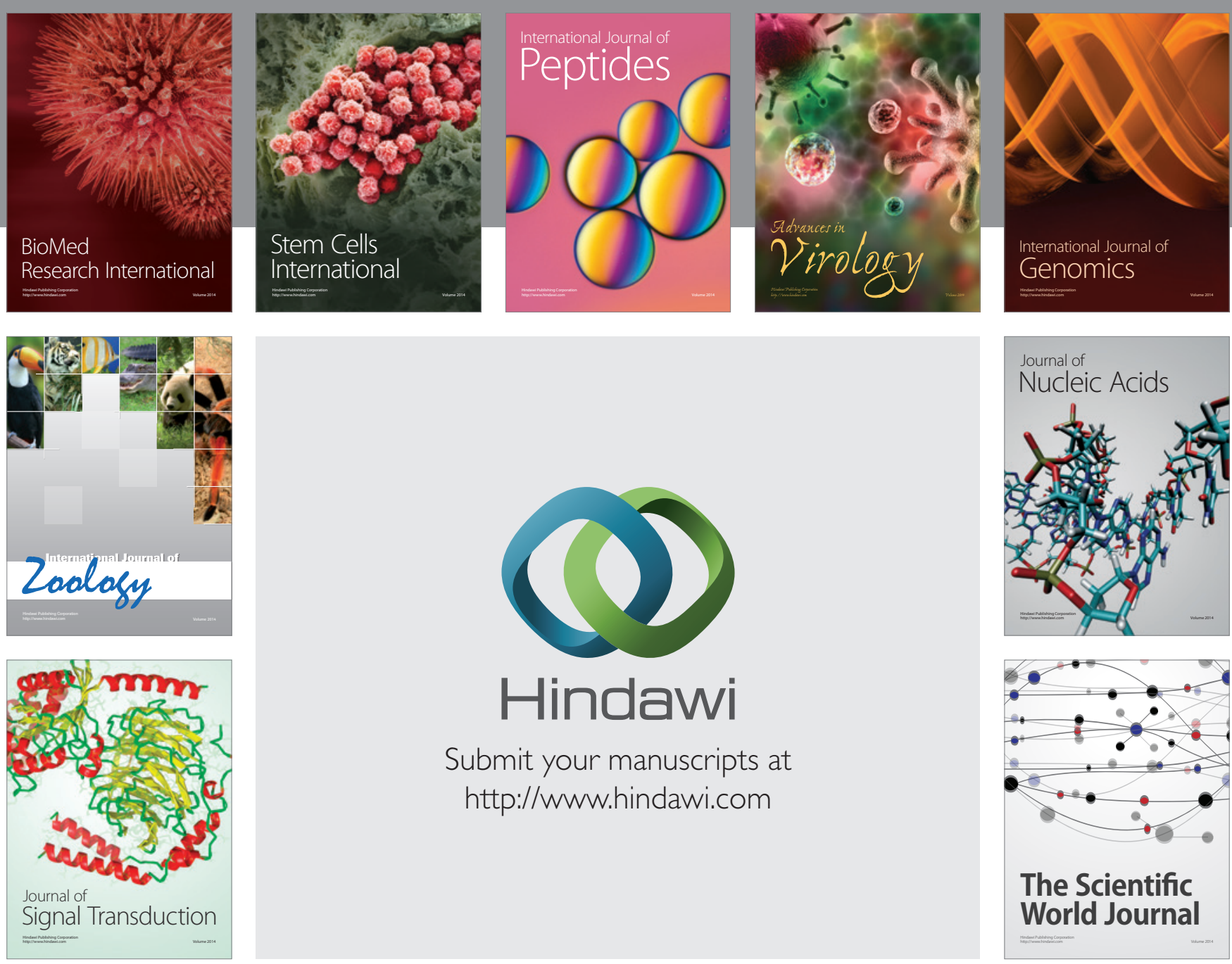

Submit your manuscripts at

http://www.hindawi.com
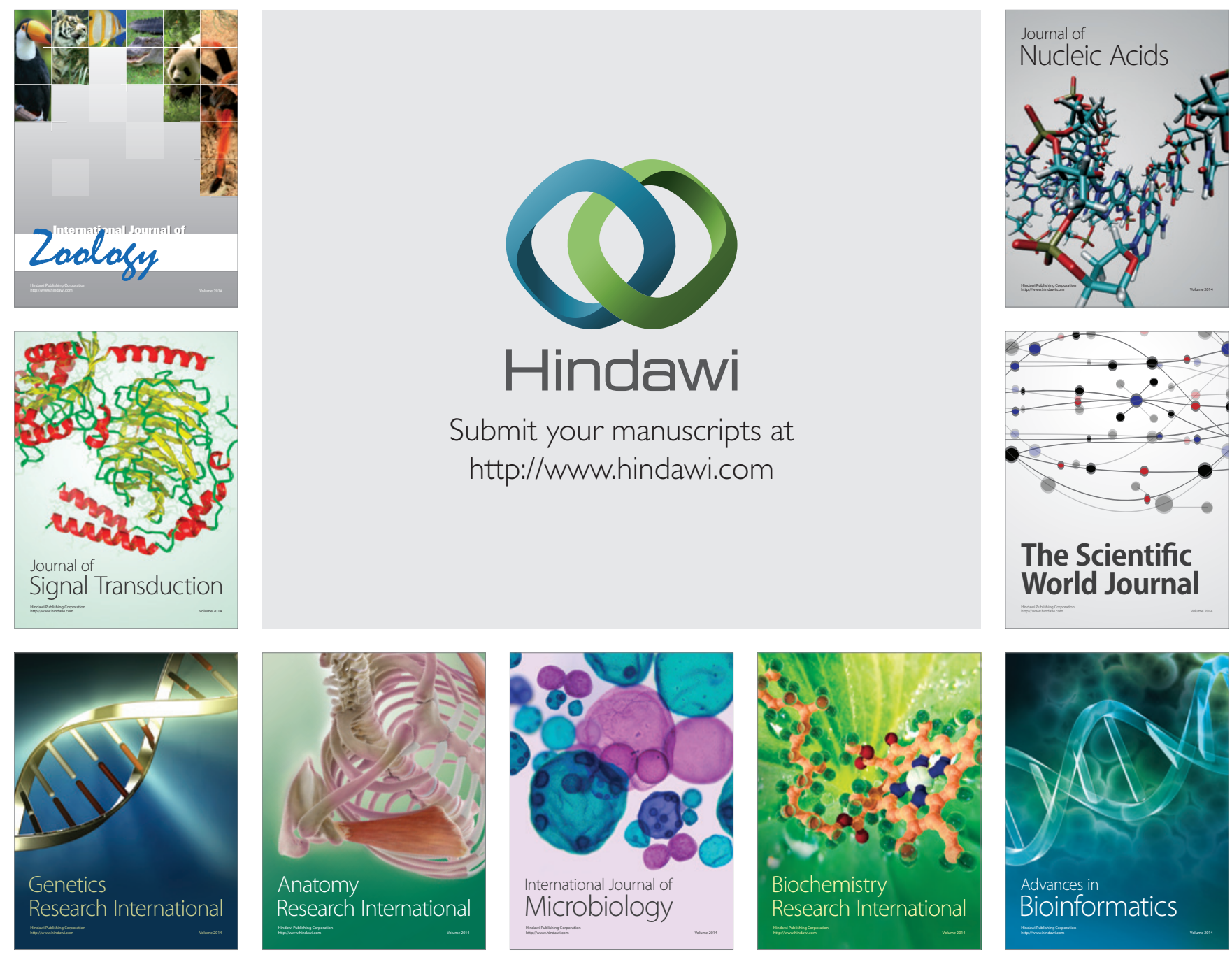

The Scientific World Journal
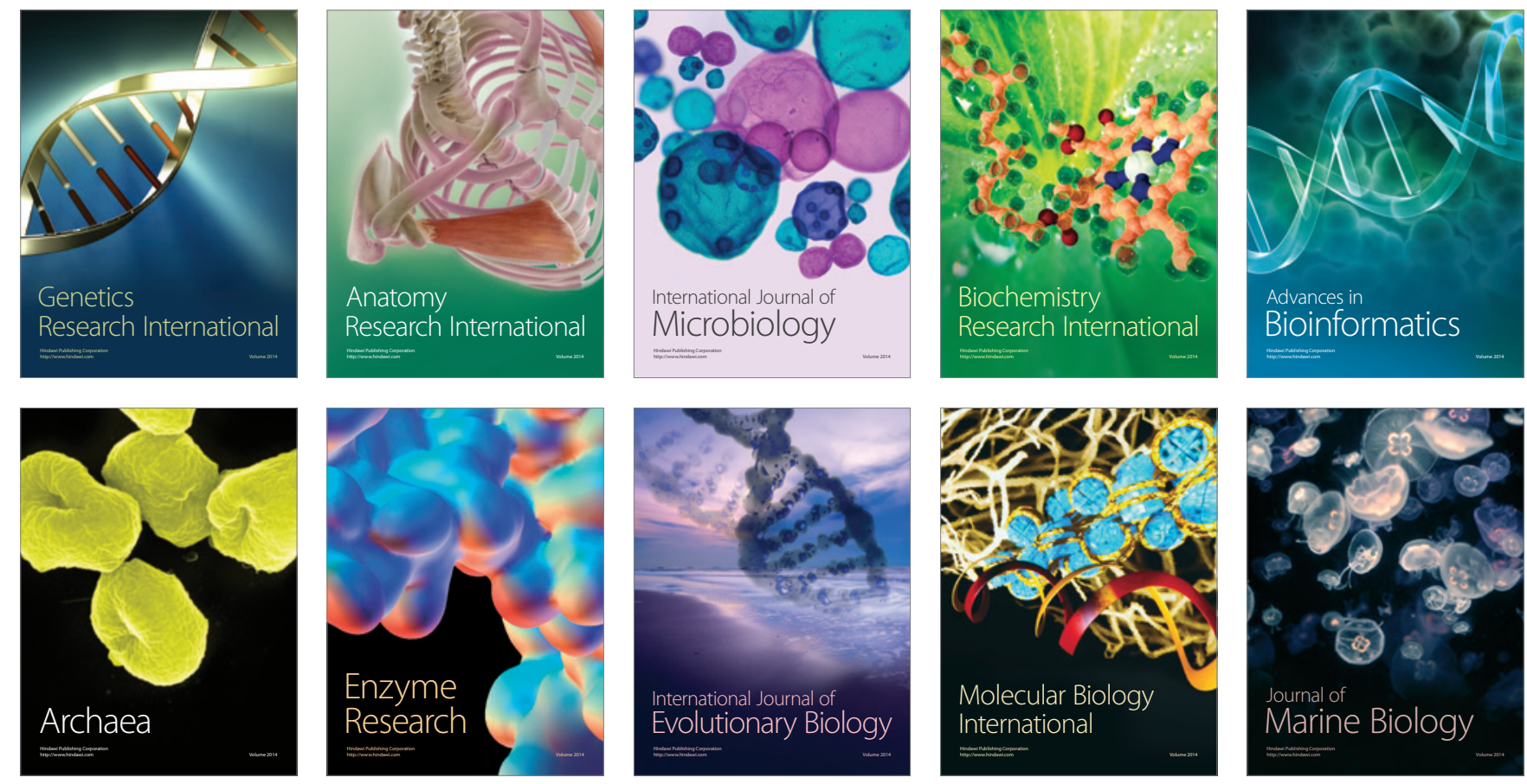